\title{
How to use orgmode for reproducible research, rough cut version 1
}

\author{
Arindam Basu ${ }^{1}$ \\ 1 University of Canterbury
}

Funding: The author(s) received no specific funding for this work.

Potential competing interests: The author(s) declared that no potential competing interests exist.

\begin{abstract}
In this article we discuss how to use orgmode for writing and weaving data analysis.
\end{abstract}

\section{A brief tutorial on org-mode: introduction}

Org-mode is a notetaking, time-management, and content authoring and thinking tool for emacs, a free and open source text editor, as a matter of fact, almost an operating system in itself that uses elisp programming language. However, you will not need to know anything aout programming languages to use Emacs. In order to use org-mode, while it can be used in any text editor, it is best to first install Emacs and then use org-mode.

Here are some use cases how orgmode can be used:

- Use org-mode as an outliner.

- Use org-mode as a planner

- Use org-mode to conduct data analyses

- Use org-mode to weave data analyses with paper writing

- Use org-mode to write academic documents

- Then export that document to various formats after parsing with pandoc, a universal document parser.

- You can also upload the org document on a git repository such as github and can share the document widely with text and code.

This implies that in itself and with pandoc, with orgmode, you have a truly reproducible research tool. Besides, if you provide the document name to pandoc command, you can convert the document to other formats and get published in formats such as Word/LaTeX and so on. You can even construct pipelines to write once and get outputs many times and in many formats. For example, you can write in org and then use pandoc to convert the document from org to markdown and then use the markdown document to be exported further. Or, you can write the document in org mode and then use pandoc to convert the document to latex and then use something like overleaf to convert the document to latex and pdf and use 
it that way. Or you can transform the document to word and then further process it.

In this tutorial, my aim is to write the simplest workflow that we can use to work with emacs and org-mode to write data-driven documents that can be converted to a number of different formats. A popular format is to write in jupyter notebooks.

\section{Mechanics of content authoring in orgmode}

\section{Adding headers and converting different types of headers}

You add header levels by adding an asterisk at the beginning of a line. The number of asterisks will determine the level of headers you want to insert. A second level header will be nested within a first level header. I usually do not use more than two levels of headers and that seems to work well for me. YMMV. By default, orgmode does not number the headers. Suppose you have written a large document or you are writing a large document and you want to jump from one header to another. You do C-c C-n which is the next heading. Or C-c C-p to jump to the previous heading or if you want to jump one level up, then you do C-c C-u. If you do C-c C-j, then emacs will ask you to move the cursor to a particular header and then hit enter and the header with it content will open up. This is referred to as drawer in org-mode.

\section{How to add headers}

If you do M-RET or press the alt key and enter, then it will insert a header at the same level next to the header in which you are working. But if you want to insert a new header BEFORE, then you will take the cursor to the beginning of the header and then hit M-RET and it will insert a new header of the same level but above the current header. Same thing if you do C-RET, that is hit control an enter at the same time. If you want to convert a second level header to a first level header, you do M-S-LEFT that is alt key plus shift key plus left key. Likewise, if you want to convert a second level header to a third level header, you do MS-> If you want to move your headers up and down, then do M-up or M-down. This will move the header and the associated content up or down depending on what you did.

\section{Cutting and pasting}

There is no concept of selecting a chunk of text and then cutting or pasting. Pasting in Emacs world is referred to as "yanking" and therefore the symbol is y. Cutting and copying are two different things. In copying you copy the content in the header with C-c C-x M-w, this will leave the text as is and then copy it to something called a "kill ring". On the other hand, if you want to cut the text, you do C-c C-x C-w and it will 'cut' the text and place it in the kill ring. Then, wherever you want, you do C-c C-x C-y.

\section{What if you want a line to be converted to a heading?}

Place the cursor in the beginning and hit M-RET. Basically, wherever you want the heading to begin, place the cursor there and hit M-RET and it will insert the heading in the same level. The same effect if you write a single line and hit C-C * and it will create a subheading. So same level heading is M-RET, subheading is Cc*

This line was converted to a header by c-c star 
I was a headline once, $\mathrm{C}-\mathrm{c} *$ turned me to a simple line

These are lines

This is a second line

All these were turned into headers by c-c* by selecting them first of course

\section{What if you want a line to be converted to a heading?}

Place the cursor in the beginning and hit M-RET. Basically, wherever you want the heading to begin, place the cursor there and hit M-RET and it will insert the heading in the same level.

Visibility cycling

using tab and shift-tab, the headers can be cycled to be visible. So, if you hit tab next to a header, that header will be shown and the document will fold up. If that header contains any text associated with it or nested headers, then you will see an ellipsis in the form of three dots to the left of this header. This is ONLY going to work if you place your cursor next to a header, it is not going to work in a paragraph like where you are reading it. So the way it works is something like as follows. You place the cursor next to the header and then hit tab key. The header only shows and the content of the header (known as tree) hides itself. Then you hit tab again and the header with the content shows up. This is useful when you are editing a large document where you do not want to be distracted with too many headers and you only want to work on some part of the document, not all of it. This works like a toggle button.

Writing tables

Adding images to orgmode documents

Adding hyperlinks

Adding citations

Adding codes

Exporting orgmode documents to other formats

\section{Visibility cycling}

using tab and shift-tab, the headers can be cycled to be visible. So, if you hit tab next to a header, that header will be shown and the document will fold up. If that header contains any text associated with it or nested headers, then you will see an ellipsis in the form of three dots to the left of this header. This is ONLY going to work if you place your cursor next to a header, it is not going to work in a paragraph like where you are reading it. So the way it works is something like as follows. You place the cursor next to the header and then hit tab key. The header only shows and the content of the header (known as tree) hides itself. Then you hit tab again and the header with the content shows up. This is useful when you are editing a large document where you do not want to be distracted with too many headers and you only want to work on some part of the document, not all of it. This works like a toggle button. 


\section{Concept of a sparse tree}

So let's say we are working on a large document and I just want to work on this particular segment where I am fully immersed. I do not want to see anythiing else. Sparse tree will enable that. To enable that, use C-c l

\section{Writing paragraphs}

\section{Copying and pasting or cutting and pasting}

\section{Writing lists}

Start with a dash sign, so

first item

$\square$ this checkbox was inserted with M-S-RET

test

test 2 this was done with first typing 1. then M-RET

and this with M-RET and so on

third item was place ABOVE second item with M-up

second item

third item again was indented

then unindentted with

with $\mathrm{M}->$ indented again

and M-RET inserted this in the same level

I was a list item once, C-c * turned me to a header

First item

Second item

Third item

the following is an unsorted list

Fifth item

First item

Third item

But doing a $\mathrm{C}-\mathrm{C}^{\wedge}$ sorted them to an alphabetically sorted lis

And then to get out of the list, hit RET twice

\section{Concept of Blocks}

HashPlusBEGIN and HashPlusEND begins and closes blocks. Usually used for codes etc.

\section{Tables}

Start with a | symbol to begin a table

Start a table with C-c |

Then use | as a column separator 
Then use |- as a horizontal line

This will turn the first row a header row

Put cell contents between | and |

Realign table with RET or TAB or C-c C-c

Within the table, TAB moves to next cell

Within the table RET moves to next row

Put the cursor outside the table and RET to end the table

\begin{tabular}{|l|l|l|}
\hline Exposure & Cases & Controls \\
\hline Exposed & 120 & 20 \\
\hline Non-exposed & 60 & 100 \\
\hline Total & 180 & 120 \\
\hline
\end{tabular}

How to edit tables

\begin{tabular}{l|l|l|l|l}
\hline What to do & Instructions \\
\hline Sort table & C-c^ \\
\hline Move between columns & M-a or M-e \\
\hline Move row down & M-DN \\
\hline Move current columnn to left or right & M-> or M-<- \\
\hline Insert new column to the left & M-S-> \\
\hline Delete column & M-S-<- \\
\hline Insert row above the current & M-S-DN \\
\hline Move row up & M-UP \\
\hline Delete Row & M-S-UP \\
\hline Insert horizontal line & C-C - \\
\hline Insert horizontal line and move cursor & C-c RET \\
\hline
\end{tabular}

Other miscellaneous things with tables 


\begin{tabular}{l|l}
$\begin{array}{l}\text { What do you want to do } \\
\text { Copy table region }\end{array}$ & Instructions \\
\hline Cut table region & C-c C-x M-w \\
\hline Paste region & C-c C-x C-y \\
\hline Split table & M-RET \\
\hline Sum numbers & C-c + then C-y \\
\hline Copy Down & S-RET \\
\hline Edit in separate window & C-c ` and finish with C-c C-c \\
\hline Import tab separated table & M-x org-table-import \\
\hline Convert a region into table & Select then C-c \\
\hline Export Table & M-x org-table-export \\
\hline For long tables, display header & M-x org-table-header-line-mode \\
\hline Transpose Table & M-x org-table-transpose-table-at-point
\end{tabular}

\section{Convert a region to table}

First type space separated data in a region

Select the region

Type C-c |

The following table was drawn on the basis of the above notes

\begin{tabular}{l|l} 
Student & test $_{\text {results }}$ \\
Tess & 100 \\
\hline Kim & 200 \\
\hline
\end{tabular}

\section{Transpposition of a time dependent table e.g.}

Write a space separated table

Then put the cursor in first row first column

Type M-x org-table-transpose-table-at-point

The following table was produced with the above steps

\begin{tabular}{|l|l|l|}
\hline $\begin{array}{l}\text { Individual } \\
\text { Score }_{\text {t1 }}\end{array}$ & 100 & 99 \\
\hline Score $_{\text {t2 }}$ & 120 & 98 \\
\hline Score $_{\text {t3 }}$ & 130 & 49 \\
\hline
\end{tabular}

The org table mode can be turned on with $M-x$ orgtbl-mode

\section{Using the table as a spreadsheet}

The table can be used as a spreadsheet. In order to do so, it is useful to use the concepts of references.

Take the following table 


\begin{tabular}{l|l|l|l|}
\hline Exposure & Cases & Controls & Total \\
\hline Exposed & 120 & 40 & 160 \\
\hline Non-exposed & 40 & 80 & 120 \\
\hline Total & 160 & 120 & 280 \\
\hline
\end{tabular}

Let's say we want to work on this as a table

If you want to turn on/off grid display, do C-c $\}$

If you want to find out coordinate of a grid, C-c ?

You can find the reference of a cell with C-c ?

Rule is: @ROW\$COLUMN

$@ 0 \$ 0$ refers to the current cell

@2 implies second row of current column

$\$ 1$ implies first column of the current row

Ranges are indicated with beginning .. end

The beginning and ends are INCLUDED

$\$ 1 . . \$ 3$ means the row elements of first, second, and third columns

Otherwise, indicate the row with @ symbol, so

$\$ 1 . . @ 2 \$ 3$ would mean from the first column to second row of third column

These range references return a VECTOR of values

Feed them into vector functions

If you want to reference another table, set a name to it, by:

$\{=$ org $\}$ \#+NAME: Name of the table

After writing the formula below the table with:

$\{=$ org $\} \#+$ TBLFM: <expression>, place cursor on the line and

Do, C-c C-c

You can write more than one formulae

If you do so, place the cursor next to each line and do C-c C-c

\section{Writing the formula}

A formula can be any algebraic expression

Example: vsum(@2..@3) will calculate the vertical sum

Use \#+TBLFM: write the formula

\section{How to insert hyperlinks to a document}

Let's say we want to place a link to an external resource such as Medium or Curvenote. We will write like this:

\section{Medium Arin's page}

Org will turn the link to a blue hyperlink

https://www.curvenote.com 
Org will turn the page to a blue hyperlink for Curvenote

But this time it leaves the URL as is as we did not provide a description

For internal links, we can do similar things with:

A section with Concept of drawers

Write the header between two squared brackets

Otherwise, for tables, do something like \#+NAME: somename and then

Add the somename between the square brackets, so

longtable will lead to the long table

You can also insert link with C-c C-I, so

NixOs webpage was inserted with this keystroke

\section{Concept of drawers}

Hiding information so use ::Draw1:: Then write something and then ::END:: ends the drawer

\section{Orgmode as productivity tool}

Orgmode can be used to store ideas and productivity stuff while writing, includng todo items and logging processes.

Create a to do item with headline with TODO keyword, or

$\mathrm{C}-\mathrm{c} \mathrm{C}-\mathrm{t}$

The following todo item was created that way

Rotate between TODO, DONE, and Plain header with C-c C-t

Or, do S-> or S-<- shift right or left

Once you have done that, and entered a few stuff, then

Use C-c / to use it as a sparse tree (see Concept of a sparse tree )

Add a new todo entry below the present one with S-M-RET

The sparse tree will show the todo items, not the done ones

We can add something like INPROCESS before DONE, so we modify

the $\sim$ /.emacs.d/init.el file with

(setq org-todo-keywords '((sequence "TODO" "INPROCESS" "|" "DONE")))

Then exit Emacs and restart and revisit an org file

How to set up dependencies for todo items

This means, you list what items must be completed before

Another item can be marked as done

and so on

Record timestamp and note when change a todo state

C-u C-c C-t then C-c C-c

This is a good practice as this forces you to recognise

What you did with it! 
Prioritise tasks with \#A \#B \#C where $\mathrm{A}=$ highest priority

INPROCESS Orgmode as a productivity tool

State "INPROCESS" from "TODO" [2021-11-04 Thu 13:45]

Still learning,

TODO Complete the tutorial by today [1/2]

DONE Find out how to rename files [2/2]

\section{How to use tags in orgmode learning tutorial editing}

Headline can have tags at the end of the headline

Tags are added with :tagword:

Two or more tags are written as :tag1:tag2:

Tags have same colour as headlines

Subheadings i.e., heading level 2 from heading level 1 inherits the tags

So in the following example, "Adding images ..." has tag learning

Adding images also has two subheadings "Adding images from files" and "Adding images from websites" Adding images from websites does not have a separate tag but it can be said that this one has inherited the learning tag

You can specify tags for files so in the preamble, do

$\{=$ org $\}$ \#+FILETAGS: :learning:tutorial:

See, same as tags for headings

Tags are searchable

Search tags with C-c/ m

How to set tags:

After headline, colon tagword colon

C-c C-q

C-c C-c works ony when you are in a headline

In the preamble of your document with \#+TAGS: keyword

\section{What are properties? learning}

You can assign properties to headlines or agenda items using drawers where the drawer name is PROPERTIES between colons and assign attributes and values or keys and values. So for example an article can be assigned properties such as title, author, year as follows

* Article Collection

If you have entered properties anywhere in the document, you can search for them using the C-c / $\mathrm{m}$ and specifying the name of the property that you created. As with the tags, properties can be inherited.

\section{Using orgmode for universal idea capture productivity uses}

First, define a directory where you will keep notes 
(setq org-default-notes-file (concat org-directory "/notes.org"))

Write the above in $\sim /$.emacs.d/init.el file

So, notes.org is the directory where I am going to store my notes

Hit M-x org-capture

Select a template, for the first time it will show $t$ for todo

Write something and then hit C-c C-c

When you do it for the first time, it will ask you to create org folder, say yes

It will create /home/arin/org directory and will place a notes.org in it

We often want to capture more than todo items, for this, create a template

Template (setq org-capture-templates '(("j" "Journal" entry (file datetree " /org/journal.org") "* \%l? on \%U \%i \%a")))

\section{How to write rich text documents in orgmode?}

\section{Writing paragraphs}

At least one empty line separate paragraphs

Use blocks to insert special elements such as VERSE and QUOTE

Bold is like this, italic is like this, and underlined

verbatim and code

strikethrough

Superscript: $x^{2}$

Subscript $x_{1}$

Toggle them with C-c C-x I

Symbols, say alpha is $\alpha$ (view with C-c C-x $\backslash$ )

For equations, set \#+OPTIONS: tex: $t$ then

for single line equations, and for multiline equations,

...*equatio**n*...

Preview with \#+STARTUP: latexpreview and then C-c C-x C-I

Horizontal rules are with five dashes

Footnotes are given as [fn:1] and then [fn:1] Description

View footnotes with C-c C-x f

\section{Adding images to orgmode document}

Do the following, so:

Write a caption of the image

Give the image a name

Insert the image with [[image url]]

then view it inline with C-c C-x C-v and toggle the view 
Then view it with C-c C-x C-v

\section{Adding citations}

Before adding citations, prepare a bibtex file

Say the bibtex file is named test.bib

Add it to the preamble with \#+bibliography: test.bib in the block

Wherever you want to insert, type M-x org-cite-insert then type the first letter of the citation key or something

Then hit tab key

It will show the matched name, accept it with RET RET

Or manually with [cite: @citation_id]

Make sure you have orgmode version 9.5 or above

find out with M-x org-version

Egger wrote @Egger1997-wq

\section{Adding and evaluating codes}

Codes can be added with blocks

Codes can also be evaluated that way

this is a test

But that was a trivial example. We will show two examples; one for $\mathrm{R}$ and the other for python.

What to do

Start a block with \#+begin_src R (or Python) ... \#+end_src

Write your code

Place cursor witin the code block

C-c C-c

Here is an example with $\mathrm{R}$

$x<-2$

$y<-9$

$\operatorname{print}(x * y)$

$x=1$

\section{return $x$}

